\title{
Fast Directional Energy Interchange Used in MCMC-based Autonomous Decentralized Mechanism toward a Resilient Microgrid
}

\author{
Yusuke SAKumoto $^{1, a)}$ ItTetsu TANiguchi $^{2, b)}$ \\ Received: March 26, 2019, Accepted: October 3, 2019
}

\begin{abstract}
In order to utilize renewable energy effectively, the generated surplus energy should be stored in batteries, and transferred to distant places with high demand in a microgrid. As a scalable mechanism for such energy transfer (energy interchange), we proposed an autonomous decentralized mechanism (ADM) based on Markov Chain Monte Carlo (MCMC), and clarified that our ADM accomplishes the global objective to quickly supply energy appropriately for energy demand all over the microgrid. In this paper, toward a resilient microgrid, we propose a method of directional energy interchange used in our ADM. We first design a method of the directional energy interchange to be able to quickly transfer energy in an appropriate direction on the basis of the advection-diffusion equation used in physics. Then, we investigate the performance of the proposed method through a simulation experiment considering energy shortage and emergency situations. Simulation results show that the proposed method (a) can quickly supply energy from a traditional centralized grid to a microgrid under energy shortage situations, and (b) can quickly gather distributed energy to a specific place (e.g., safe shelter) under emergency situations.
\end{abstract}

Keywords: autonomous decentralized control, energy transfer, microgrid, renewable energy, resilience

\section{Introduction}

Renewable energy resources (e.g., sunlight and wind) are hopefully alternatives to conventional fuels (e.g., fossil fuel and nuclear fuel). The generation using renewable energy is well known as low carbon emission, but its output is usually small scale and unstable. Hence, renewable energy generators have difficulty supplying the generated energy appropriately for the energy demand. In order to promote the transition to renewable energy, it is necessary to develop a mechanism to perform such an energy supply using renewable energy as much as possible.

In Ref. [1], a microgrid is introduced as an electricity system with a network interconnecting distributed batteries, electronic loads, and micro-scale generators (e.g., photovoltaic array and wind turbines). A microgrid not only works independently from a traditional centralized grid (hereafter referred to as macrogrid) and other microgrids, but also cooperates with them during an emergency. In microgrids, the generated energy is stored in batteries in order to absorb the time variation of energy supply and demand. Additionally, surplus energy in a battery is transferred to distant electric loads with high demand in order to absorb the difference among energy demands of electric loads. In our work, we call such energy transfer energy interchange. The energy interchange in microgrids is also a key technology to improve renewable energy's ease of use. Facing the energy transition, mi-

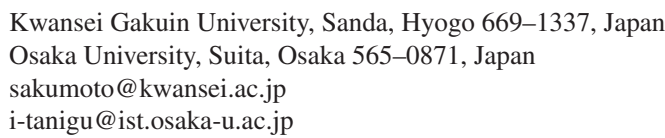

crogrids should be able to hold enormous devices distributed in a wide area. Hence, microgrids require a scalable mechanism of the energy interchange so as to easily extend its area and increase its number of composition devices.

We proposed a novel autonomous decentralized mechanism (ADM) based on Markov Chain Monte Carlo (MCMC) for the energy interchange in large-scale and wide-area microgrids [2]. In our proposal, a microgrid is composed of autonomous electricity subsystems (AESs), which work asynchronously for scalability. An AES means a unit of microgrid. AES, for instance, corresponds to the localized electricity system in a building (e.g., a home). AESs are interconnected like a mesh network shown in Fig. 1. We assume that each AES has a battery, and can transfer its own energy to another AES with an arbitrary amount. The energy transmission between both AESs is controlled by them. In our ADM, each AES autonomously stores energy in its own battery, and performs energy interchange to another AES according to its energy demand. The autonomous decision of AESs was designed on the basis of the MCMC so as to accomplish the global objective to quickly supply energy appropriately for energy demand all over the large-scale mesh network. However, there remain important works in our research. Namely, we have not fully discussed the necessary abilities and functions of our ADM against inevitable crisis events (e.g., abnormal weather and disaster).

We assume that our ADM works on the microgrid with a virtual power plant (VPP) [3] that virtually aggregates the distributed generators, batteries, and electronic loads. In the microgrid with a VPP, each node is virtually connected by ICT technology. The 


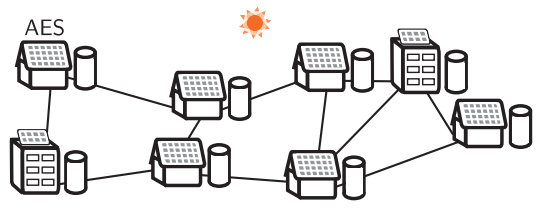

Fig. 1 Mesh network in a microgrid.

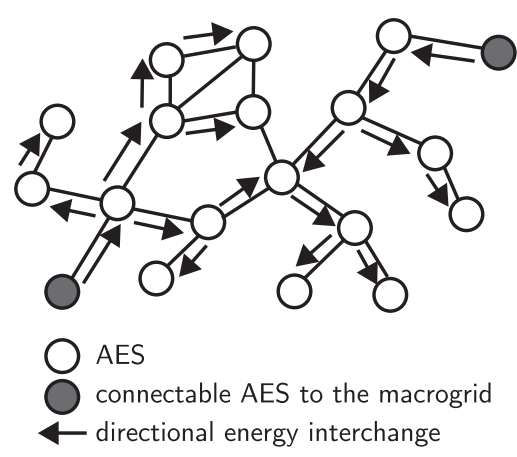

Fig. 2 Directional energy interchange in a direction away from AESs connecting a macrogrid.

node in the VPP corresponds to AES in our ADM. The difference between the macrogrid and the microgrid with a VPP is the calculation of the energy interchange amount based on information gathered via virtual connections of nodes before transferring energy physically. The microgrid with a VPP may take longer to achieve the supply-demand balance than the macrogrid that is totally physically optimized. However, VPPs realize highly flexible energy management (e.g., demand response), and some projects [3], [4], [5], [6], [7] put VPPs into practical use. From these reasons, we assume the proposed mechanism works on a microgrid with a VPP.

In this paper, we focus on the events that require resilience to microgrids, and discuss what kind of energy interchange is needed to have the resilience. Note that the term "resilience" means that the ability to quickly recover from and withstand a difficult situation [8]. Abnormal weather and disaster will definitely occur, and require the resilience to microgrids to be readily available against such inevitable crisis events. If the weather is abnormal, generators using renewable energy may not supply energy to be able to satisfy energy demand in a microgrid. For such an energy shortage situation, a macrogrid should fill the energy shortage in a microgrid. However, connectable AESs to the macrogrid are limited by geographical conditions. Hence, it is necessary to quickly supply energy in a direction away from the connectable AESs as in Fig. 2. When a disaster (e.g., earthquake) occurs, people evacuate to a safe shelter, and energy should be preferentially supplied there. For such an emergency situation, energy in a microgrid should be quickly gathered at the safe shelter. Hence, it is necessary to quickly supply energy in the direction to a high priority AES from other AESs as in Fig. 3. According to the above discussion, in order to have the resilience, a mechanism in microgrids should perform directional energy interchange to recover from and withstand energy shortage and emergency situations.

In this paper, toward resilient microgrids, we propose a method to realize the directional energy interchange that enables the MCMC-based ADM [2] to quickly transfer energy in an appropri-

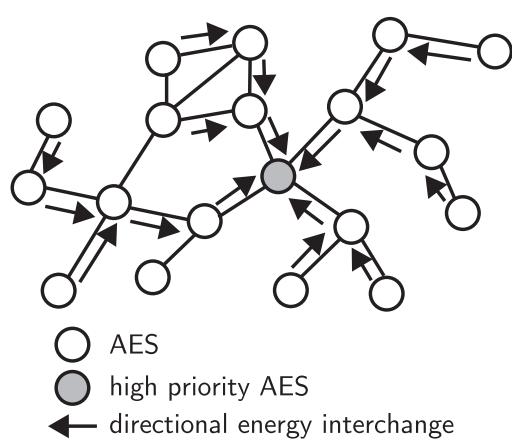

Fig. 3 Directional energy interchange in a direction to a high priority AES from other AESs.

ate direction. We first design the directional energy interchange based on the advection-diffusion equation, and then investigate the effectiveness of the proposed method through a simulation experiment considering energy shortage and emergency situations. In the proposed method, we assume that some AESs can be connected to a macrogrid, and can supply energy to other AESs in the microgrid. We clarify that the proposed method can quickly supply energy from a macrogrid to a microgrid under an energy shortage situation, and quickly gather distributed energy to a specific AES (e.g., safe shelter) under an emergency situation. In the conference paper [9], we introduced the proposed method briefly. This paper is the extended version of the conference paper [9], and includes (a) the detailed explanation of the proposed method and (b) advanced evaluation with a time-varying model also reported in Ref. [10].

This paper is organized as follows: Section 2 introduces the related works, and discusses the characteristics of our works by comparison with other works. Section 3 explains the system model. Section 4 describes the MCMC-based ADM for energy interchange. Section 5 shows the method for fast directional energy supply. Experimental results are described in Section 6. Section 7 concludes this paper, and discusses the future work.

\section{Related Works}

\subsection{Microgrid Projects for Realizing the Energy Inter- change}

Several projects [6], [7] are gradually putting a microgrid with an electricity network as in Fig. 1 into practical use. A nonprofit organization [6] proposed the concept of the community microgrid that utilizes energy interchange in a microgrid, and is demonstrating the community microgrids in California and New York. An energy technology company [7] is building a peer-to-peer energy market to accomplish the energy interchange in the microgrid in New York. If such microgrids can cover the entire city, they would promote the transition to renewable energy from conventional fuel. However, in order to realize the full coverage of a city with microgrids, it is necessary to develop a scalable mechanism so as to easily extend its area and increase its number of composition devices. Our work aims to realize such a scalable mechanism.

\subsection{A Mechanism of the Energy Interchange}

Some previous studies [11], [12] proposed a mechanism of the 
energy interchange in microgrids with an AES mesh network similar to our work. In Ref. [11], the authors designed the internetlike mechanism of the multi-hop energy interchange to transfer energy between an AES pair in a mesh network. In Ref. [12], the authors supposed a virtual market of energy interchange among AESs. In the virtual market, each AES trades energy using the algorithm designed by machine learning, and transfers energy to another AES according to the trading results. Unlike the previous works, our ADM does not depend on any complex mechanisms (i.e., the multi-hop routing and the virtual market), and works only using the light-weight autonomous action to be able to supply energy appropriately for energy demand all over the mesh network. Hence, our ADM is more scalable than those of the previous works.

\subsection{The Resilience of Electricity Systems}

The resilience is important for electricity systems because they need high reliability and availability. Hence, there are many studies and projects focused on the resilience [13], [14], [15], [16], [17], [18], [19]. These works are divided into two categories. The works [13], [14], [15], [16], [17] in the first category improved the resilience of existing electricity systems by utilizing microgrids. The Sendai microgrid [14] is built for the electricity system of Tohoku Fukushi University. According to the report [15], the Sendai microgrid at Tohoku Fukushi University operated for 2 days without the energy supply of the macrogrid when hitting the 2011 Great East Japan Earthquake. In the second category, the works [18], [19] dedicate the development of a mechanism to improve the resilience of microgrids. This paper is one of the second category works. We will improve the resilience of microgrids with our ADM.

\subsection{The Consensus Problem}

The MCMC-based ADM is a solution to the consensus problem in a mesh network. The consensus problem is a fundamental problem that discusses that the values of each node in a network converge to a common value, and appears in various engineering subjects [20], [21]. Many methods solve the consensus problem with linear dynamics, but our method utilizes non-linear dynamics for fast convergence. We have only applied our method to the energy interchange in microgrids, but it is expected to be applicable to other subjects related to the consensus problem.

\section{System Model}

We use the system model, which has also been used in Ref. [2]. The system model describes the energy interchange among AESs. In the system model, each AES interconnects with other AESs like a mesh network shown in Fig. 1. We denote the mesh network by $G(V, E)$ where $V$ is the set of AESs, and $E$ is the set of links among AESs. $E$ has the link between an AES pair $(i, j)$ iff an AES $i$ can perform energy interchange to/from AES $j$. Table 1 shows the definition of symbols used in the system model. The system model assumes that (a) any energy loss in energy transformation and interchange of AESs can be ignored, and (b) the rate-of-change in battery charging is not limited. These assumptions are not feasible, but are necessary to focus on the effect of
Table 1 Definition of symbols.

\begin{tabular}{c|l}
\hline \hline symbol & definition \\
\hline$G$ & mesh network of AESs in a microgrid \\
$V$ & set of AESs in mesh network $G$ \\
$E$ & set of links between a pair of AESs in mesh network $G$ \\
$n$ & number of AESs in mesh network $G$ \\
$\alpha_{i}$ & set of adjacent AESs of AES $i$ \\
$v_{i}(t)$ & subset of adjacent AESs for which AES $i$ should perform \\
& directional energy interchange at time $t$ \\
$g_{i}(t)$ & energy generation rate of AES $i$ at time $t$ \\
$c_{i}(t)$ & energy consumption rate of AES $i$ at time $t$ \\
$\gamma_{i}$ & battery capacity of AES $i$ \\
$b_{i}(t)$ & remaining battery amount of AES $i$ at time $t\left(0 \leq b_{i}(t) \leq \gamma_{i}\right)$ \\
$\theta_{i}$ & target remaining battery amount of AES $i\left(0 \leq \theta_{i} \leq \gamma_{i}\right)$ \\
$d_{i}(t)$ & sufficiency level of AES $i$ at time $t$ \\
$r_{i \rightarrow j}(t)$ & energy interchange rate from AES $i$ to AES $j$ at time $t$ \\
$r_{i \rightarrow j}^{\mathrm{MC}}(t)$ & energy interchange rate based on MCMC from AES $i$ to \\
$r_{i \rightarrow j}^{\text {ad }}(t)$ & AES $j$ at time $t$ \\
& energy interchange rate with the advection diffusion from \\
$\kappa_{\mathrm{diff}}$ & AES $i$ to AES $j$ at time $t$ \\
$\kappa_{\mathrm{MC}}$ & diffusion coefficient of the MCMC-based ADM \\
$\kappa_{\mathrm{ad}}$ & MCMC control parameter of the MCMC-based ADM \\
$\Delta T_{r}$ & advection coefficient of the proposed method \\
\hline & interval of recalculating $r_{i \rightarrow j}(t)$ \\
\hline
\end{tabular}

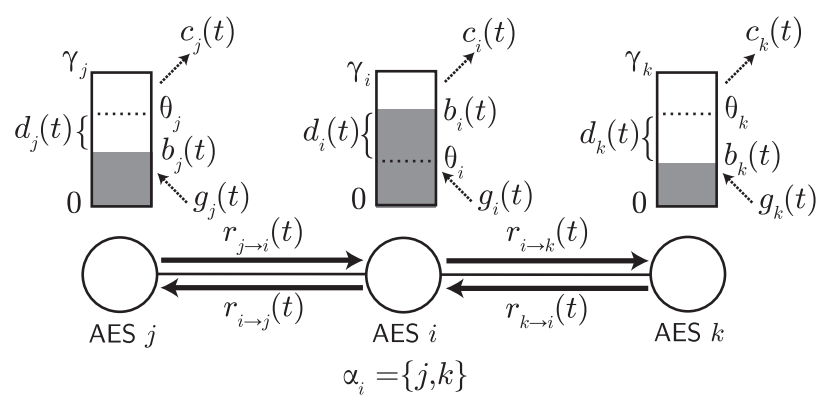

Fig. 4 System model.

energy interchange among AESs, and investigate the characteristics of our energy interchange mechanism. The system model with the energy loss and the limitation of rate-of-change will be considered in future work.

In a situation without energy transformation and transmission loss, the evolution of remaining battery amount $b_{i}(t)$ in AES $i$ $\left(0 \leq b_{i}(t) \leq \gamma_{i}\right)$ is simply given by

$$
\frac{\mathrm{d} b_{i}(t)}{\mathrm{d} t}=g_{i}(t)-c_{i}(t)+\sum_{j \in \alpha_{i}}\left(r_{j \rightarrow i}(t)-r_{i \rightarrow j}(t)\right),
$$

where $g_{i}(t)$ and $c_{i}(t)$ are the energy generation and consumption rates of AES $i$ at time $t$, respectively. Note that $c_{i}(t)$ represents instantaneous energy demand of AES $i$. In Eq. (1), $\alpha_{i}$ is the set of adjacent AESs of AES $i$ in mesh network $G$, and $r_{i \rightarrow j}(t)$ is the energy interchange rate from AES $i$ to an adjacent AES $j \in \alpha_{i}$. AES $i$ recalculates energy interchange rate $r_{i \rightarrow j}(t)$ every $\Delta T_{r}$. In Fig. 4, we illustrate the system model. In this figure, remaining battery amount $b_{i}(t), b_{j}(t)$, and $b_{k}(t)$ are changing with not only its energy generation and consumption, but also the energy interchange among AESs. We denote the battery capacity of AES $i$ by $\gamma_{i}$.

In general, each AES has different energy demand than other AESs. To represent achievement of energy supply appropriately for energy demand in an AES, we introduce sufficiency level $d_{i}(t)$ of AES $i$ by

$$
d_{i}(t):=b_{i}(t)-\theta_{i},
$$


where $\theta_{i}$ is the target remaining battery amount of AES $i$. If $b_{i}(t) \geq \theta_{i}$, the energy demand of AES $i$ is satisfied. $\theta_{i}$ is set by the administrator of AES $i$. In the system model, we assume that $\theta_{i}$ is time-invariant in order to investigate the characteristics of our energy interchange mechanism.

AES $i$ can know whether its an adjacent AES $j \in \alpha_{i}$ needs more energy by the observation of $d_{j}(t)$. If $d_{i}(t)=d_{j}(t)$ for all AES pairs $(i, j)$, the energy supply would be achieved appropriately for the energy demand of AESs at time $t$. The MCMC-based ADM performs energy interchanges to equalize sufficiency levels of all AESs.

\section{MCMC-based Autonomous Decentralized Mechanism of Energy Interchange}

\subsection{Autonomous Decision}

To equalize sufficiency levels of all AESs with the MCMCbased ADM [2], AES $i$ calculates energy interchange rate $r_{i \rightarrow j}^{\mathrm{MC}}(t)$ to an adjacent AES $j \in \alpha_{i}$ using

$$
r_{i \rightarrow j}^{\mathrm{MC}}(t)=\kappa_{\mathrm{diff}}\left[1-\kappa_{\mathrm{MC}} f_{i \rightarrow j}\left(d_{i}(t), d_{j}(t)\right)\right] d_{i}(t)
$$

where $\kappa_{\mathrm{diff}}$ and $\kappa_{\mathrm{MC}}$ are positive parameters that determine equalizing speed of sufficiency levels ${ }^{* 1}$. In Eq. (3), $f_{i \rightarrow j}\left(d_{i}(t), d_{j}(t)\right)$ is given by

$$
\begin{aligned}
& f_{i \rightarrow j}\left(d_{i}(t), d_{j}(t)\right) \\
& \quad=\left|d_{j}(t)-d_{i}(t)\right|\left[\operatorname{sgn}\left(d_{i}(t)\right)\left(d_{j}(t)-d_{i}(t)\right)\right]^{+},
\end{aligned}
$$

where $\operatorname{sgn}(x)$ is the sign of $x$, and $[x]^{+}=\max (0, x)$. According to Ref. [21], $\kappa_{\text {diff }} \Delta T_{r}$ should be set in $0<\kappa_{\text {diff }} \Delta T_{r}<$ $1 / \max \left(\left|\alpha_{i}\right|\right)$ for all $i$ for the stability because $r_{i \rightarrow j}^{\mathrm{MC}}(t)$ is updated every $\Delta T_{r}$. If $\kappa_{\text {diff }}$ is too large, the MCMC-based ADM becomes unstable. Since Eqs. (3) and (4) only depend on the information of AES $i$ and an adjacent AES $j \in \alpha_{i}$, each AES can autonomously calculate energy interchange rate $r_{i \rightarrow j}^{\mathrm{MC}}(t)$ by gathering the information of adjacent AESs.

In Ref. [2], we proved that the sufficiency levels of all AESs are equalized by repeating the energy interchange among AESs with the above autonomous decision. Equations (3) and (4) are derived on the basis of MCMC [22], which is a method to control the probability distribution of a variable in statistical mechanics model (e.g., Ising spin model [23]). In Ref. [2], we applied MCMC to control the probability distribution of the variance of sufficiency levels. If the variance is 0 , sufficiency levels are equalized. Using Eqs. (3) and (4), the probability distribution of the variance can be controlled to the Dirac's delta function, which has a very sharp peak at 0 . Hence, the autonomous decision can always equalize the sufficiency levels of all AESs with the energy interchange, and so accomplishes the global objective to quickly supply energy appropriately for energy demand all over the largescale mesh network. The advantage of using MCMC is to be able to design the autonomous decision depending on the variance of sufficiency levels. By designing it with MCMC, the strong force

*1 In Ref. [2], we describe the old version of Eqs. (3) and (4) for only $d_{i}(t) \geq 0$, so they do not have $\operatorname{sgn}\left(d_{i}(t)\right)$. The description in Ref. [2] has a typo. Equations (3) and (4) in this paper are correct. works for equalizing sufficiency levels if the variance of sufficiency levels is large, and the equalizing speed becomes fast.

The autonomous decision with Eq. (3) does not consider the limitation of batteries. Hence, overrun (i.e., $\left.b_{i}(t)>\gamma_{i}\right)$ and underrun (i.e., $b_{i}(t)<0$ ) of batteries may occur. In Ref. [2], we proposed the modified expression of $r_{i \rightarrow j}^{\mathrm{MC}}(t)$ to avoid the overrun and underrun by the energy interchange. The detail explanation is given in the section 3.3 of Ref. [2].

\subsection{Diffusion Effect}

The evolution of sufficiency level $d_{i}(t)$ with the above autonomous decision of each AES can be described with a diffusion equation. The equalizing $d_{i}(t)$ with the MCMC-based ADM comes from the diffusion effect of the diffusion equation.

In order to focus on the diffusion effect of the MCMC-based ADM, we discuss the evolution of sufficiency level $d_{i}(t)$ when generation rate $g_{i}(t)$ is equal to consumption rate $c_{i}(t) i(1 \leq i \leq n)$ for all time $t$. In this condition, the evolution of sufficiency level $d_{i}(t)$ is given by

$$
\frac{\mathrm{d} d_{i}(t)}{\mathrm{d} t}=\frac{\mathrm{d} b_{i}(t)}{\mathrm{d} t}=\sum_{j \in \alpha_{i}}\left(r_{j \rightarrow i}^{\mathrm{MC}}(t)-r_{i \rightarrow j}^{\mathrm{MC}}(t)\right) .
$$

By substituting Eqs. (3) and (4) into Eq. (5), the evolution of sufficiency level $d_{i}(t)$ is derived as

$$
\frac{\mathrm{d} d_{i}(t)}{\mathrm{d} t}=\sum_{j \in \alpha_{i}} h_{i \rightarrow j}\left(d_{i}(t), d_{j}(t)\right)\left(d_{j}(t)-d_{i}(t)\right),
$$

where

$$
\begin{aligned}
h_{i \rightarrow j}\left(d_{i}(t), d_{j}(t)\right) \\
\quad= \begin{cases}\kappa_{\mathrm{diff}}\left[1+\kappa_{\mathrm{MC}}\left|d_{j}(t)-d_{i}(t)\right| \min \left(\left|d_{i}(t)\right|,\left|d_{j}(t)\right|\right)\right] \\
\kappa_{\mathrm{diff}} & \text { if } \operatorname{sgn}\left(d_{i}(t)\right)=\operatorname{sgn}\left(d_{j}(t)\right)\end{cases}
\end{aligned}
$$

Equation (6) is the discrete diffusion equation of $d_{i}(t)$ with non-linear coefficient $h_{i \rightarrow j}\left(d_{i}(t), d_{j}(t)\right)$ on mesh network $G$. Note that Eq. (6) becomes the linear diffusion equation if $\kappa_{\mathrm{MC}}$ is set to 0 . The term with $\kappa_{\mathrm{MC}}$ generates the non-linear diffusion effect to alleviate the energy interchange for the opposite direction to equalize sufficiency levels. According to Eq. (7), if the difference $\left|d_{j}(t)-d_{i}(t)\right|$ is small, non-linear coefficient $h_{i \rightarrow j}\left(d_{i}(t), d_{j}(t)\right)$ is approximately equal to $\kappa_{\text {diff }}$. As the difference $\left|d_{j}(t)-d_{i}(t)\right|$ increases, $h_{i \rightarrow j}\left(d_{i}(t), d_{j}(t)\right)$ increases. Hence, the non-linear effect can automatically adjust the coefficient of Eq. (6) to fast decrease the difference among sufficiency levels. Because of this non-linear effect, the MCMC-based ADM can fast equalize sufficiency levels of all AESs. From Eqs. (6) and (7), it turns out that the equalized state (i.e., $d_{i}(t)=d_{j}(t)$ for all $\operatorname{AES}$ pairs $\left.(i, j)\right)$ is the only steady state of the discrete diffusion equation. In the linear diffusion equation (i.e., Eq. (6) with $\kappa_{\mathrm{MC}}=0$ ), the variance always converges to 0 . According to Ref. [2], the variance of sufficiency levels if $\kappa_{\mathrm{MC}}>0$ is always smaller than that if $\kappa_{\mathrm{MC}}=0$. Hence, in the non-linear diffusion equation (i.e., Eq. (6) with $\kappa_{\mathrm{MC}}>0$ ), the variance also always converges to 0 . Hence, we also prove that 


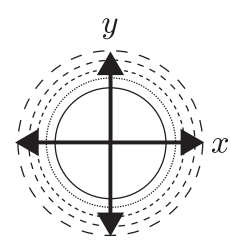

(a) diffusion (b) diffusion and advection for $x$

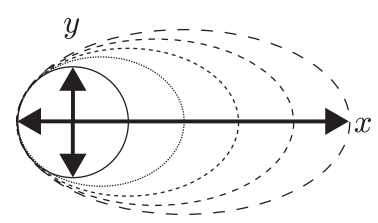

the MCMC-based ADM can always equalize $d_{i}(t)$ on the basis of the diffusion effect.

The diffusion effect of the MCMC-based ADM given by Eqs. (6) and (7) does not have strong directionality. In the MCMC-based ADM, the surplus energy is diffused uniformly to the whole of mesh network $G$. If $G$ is approximated by a twodimensional plane with continuous variables $(x, y)$, the equalizing sufficiency level $d_{i}(t)$ is essentially illustrated by Fig. 5 (a), which shows the diffusion of the surplus energy from a AES at the center position on the $(x, y)$ plane. Hence, the MCMC-based ADM would not help the diverse situations (e.g., energy shortage and emergency situations) requiring the directional energy supply shown in Figs. 2 and 3.

\section{Method for Fast Directional Energy Inter- change Based on Advection Diffusion}

\subsection{Basic Idea}

The advection-diffusion equation is used to describe the directional diffusion phenomenon of particles shown in Fig. 5 (b). By the comparison with the diffusion phenomenon, the advectiondiffusion phenomenon has an additional effect to quickly diffuse particles for a specific direction. In the example shown in Fig. 5 (b), the particles are quickly diffused for $x$-axis direction on the $(x, y)$-plane. Hence, the advection-diffusion equation is useful to design a method that enables the MCMC-based ADM to perform the directional energy interchange.

\subsection{Design}

In order to design the method for the directional energy interchange on mesh network $G$, we obtain the advection-diffusion equation for $G$. On the basis of the obtained equation, we derive the autonomous decision to perform the directional energy interchange.

The advection diffusion in the two-dimensional lattice network with discrete variable $\left(x_{i}, y_{i}\right)$ is easier to understand than that in mesh network $G$. Hence, we first discuss the two-dimensional lattice network, which is obtained by the discretization of the $(x, y)$ plane. Figure 6 shows the part of the two-dimensional lattice network. In the lattice network, each node has 4 adjacent nodes equally. Hence, it is easy to understand the advection-diffusion phenomenon in the lattice network unlike general mesh networks.

With the advection for the direction of $x_{i}$ in the lattice network like Fig. 5 (b), the linear advection-diffusion equation of $q_{i}(t)$ is given by

$$
\frac{\mathrm{d} q_{i}(t)}{\mathrm{d} t}=\sum_{j \in \alpha_{i}} \kappa_{\mathrm{diff}}^{\prime}\left(q_{j}(t)-q_{i}(t)\right)-\kappa_{\mathrm{ad}}^{\prime}\left(q_{j_{\mathrm{R}}}(t)-q_{j_{\mathrm{L}}}(t)\right)
$$

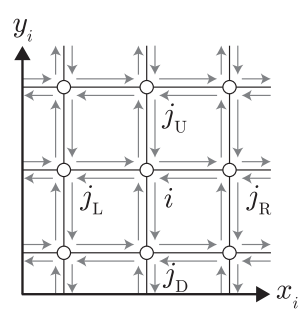

(a) bidirectional interaction of diffusion (b) directional interaction of advection for $x_{1}$

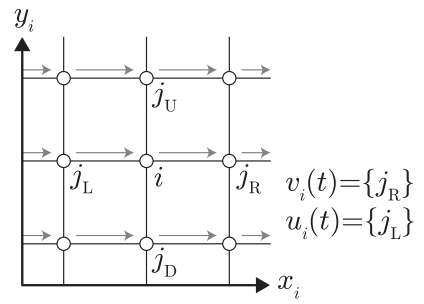

Fig. 6 Interaction for the diffusion and the advection in the $\left(x_{i}, y_{i}\right)$-lattice network.

$$
\begin{aligned}
= & \sum_{j \in \alpha_{i}} \kappa_{\mathrm{diff}}^{\prime}\left(q_{j}(t)-q_{i}(t)\right) \\
& -\kappa_{\mathrm{ad}}^{\prime}\left[\left(q_{j_{\mathrm{R}}}(t)-q_{i}(t)\right)+\left(q_{i}(t)-q_{j_{\mathrm{L}}}(t)\right)\right],
\end{aligned}
$$

where $\kappa_{\text {diff }}^{\prime}$ and $\kappa_{\text {ad }}^{\prime}$ are the diffusion coefficient and advection coefficient in the lattice network. Adjacent node set $\alpha_{i}$ of node $i$ is given by $\left\{j_{\mathrm{L}}, j_{\mathrm{R}}, j_{\mathrm{U}}, j_{\mathrm{D}}\right\}$. The term with $\kappa_{\text {diff }}^{\prime}$ in Eq. (8) describes the two-way interaction between nodes $i$ and $j$ for generating the diffusion. In Fig. 6 (a), we illustrate two-way interactions by allows. Ignoring the difference between $\kappa_{\text {diff }}^{\prime}$ and $h\left(d_{i}(t), d_{j}(t)\right)$, the term with $\kappa_{\text {diff }}^{\prime}$ corresponds to the right hand side of Eq. (6) that describes the diffusion effect of the MCMC-based ADM. On the contrary, Eq. (6) does not have a term corresponding to that with $\kappa_{\mathrm{ad}}^{\prime}$ in Eq. (8). The term with $\kappa_{\mathrm{ad}}^{\prime}$ describes the one-way interaction between nodes $i$ and $j$ for generating the advection for $x_{i}$. The one-way interactions related to node $i$ are divided into two types: incoming interaction from node $j_{L}$ to node $i$, and outgoing interaction from node $i$ to node $j_{R}$. Different combination of incoming and outgoing interactions generates different advection. In order to describe various types of the advection in mesh network $G$, we should generalize the advection-diffusion equation given by Eq. (8).

To generalize the advection-diffusion equation, we first introduce $v_{i}(t)$ and $u_{i}(t)$, which $v_{i}(t)$ and $u_{i}(t)$ are the subsets of adjacent nodes of node $i$ to perform the incoming and outgoing interactions at $t$, respectively. Since these interactions are one way, $v_{i}(t) \cap u_{i}(t)=\emptyset$. The type of the advection is determined by the combination of $v_{i}(t)$ and $u_{i}(t)$. The relation between $u_{i}(t)$ and $v_{j}(t)$ is given by $u_{i}(t)=\left\{j \mid i \in v_{j}(t)\right\} . v_{i}(t)$ and $u_{i}(t)$ must be $v_{i}(t) \subseteq \alpha_{i}$ and $u_{i}(t) \subseteq \alpha_{i}$. In the example shown in Fig. 6(b), $v_{i}(t)$ and $u_{i}(t)$ are given by $\left\{j_{\mathrm{R}}\right\}$ and $\left\{j_{\mathrm{L}}\right\}$, respectively.

Using $v_{i}(t)$ and $u_{i}(t)$, the advection-diffusion equation of sufficiency level $d_{i}(t)$ in mesh network $G$ can be derived as

$$
\begin{aligned}
\frac{\mathrm{d} d_{i}(t)}{\mathrm{d} t}= & \sum_{j \in \alpha_{i}} h_{i \rightarrow j}\left(d_{j}(t), d_{i}(t)\right)\left(d_{j}(t)-d_{i}(t)\right) \\
& -\kappa_{\mathrm{ad}}\left[\sum_{j \in v_{i}(t)}\left(d_{j}(t)-d_{i}(t)\right)+\sum_{j \in u_{i}(t)}\left(d_{i}(t)-d_{j}(t)\right)\right] .
\end{aligned}
$$

To obtain the above equation, we generalize the advection in Eq. (8) using $v_{i}(t)$ and $u_{i}(t)$, and substitute $h_{i \rightarrow j}\left(d_{j}(t), d_{i}(t)\right)$ and $\kappa_{\text {ad }}$ for $\kappa_{\text {diff }}^{\prime}$ and $\kappa_{\text {ad }}^{\prime}$, respectively. Equation (9) without the term with $\kappa_{\text {ad }}$ is the same as Eq. (6).

On the basis of the generalized advection-diffusion equation 
given by Eq. (9), we design the autonomous decision of AES $i$ for directional energy interchange. In Eq. (9), AES $i$ performs and receives directional energy interchange for adjacent AES $j$ in $v_{i}(t)$ and $u_{i}(t)$, respectively. To perform such the directional energy interchange in the MCMC-based ADM, we should add a part of the term with $\kappa_{\mathrm{ad}}$ in the expression of $r_{i \rightarrow j}^{\mathrm{MC}}(t)$. As the added expression of $r_{i \rightarrow j}^{\mathrm{MC}}(t)$, we derive the following expression of $r_{i \rightarrow j}^{\text {ad }}(t)$ that is given by

$$
\begin{aligned}
r_{i \rightarrow j}^{\mathrm{ad}}(t)= & \kappa_{\mathrm{diff}}\left[1-\kappa_{\mathrm{MC}} f_{i \rightarrow j}\left(d_{i}(t), d_{j}(t)\right)\right] d_{i}(t) \\
& -I_{j \in v_{i}(t)} \kappa_{\mathrm{ad}}\left(d_{j}(t)-d_{i}(t)\right)
\end{aligned}
$$

where

$$
I_{j \in v_{i}(t)}= \begin{cases}1 & \text { if } j \in v_{i}(t) \\ 0 & \text { otherwise }\end{cases}
$$

Note that the term with $\kappa_{\text {diff }}$ in Eq. (10) is the same as the right side of Eq. (3). The proposed method uses the expression of $r_{i \rightarrow j}^{\text {ad }}(t)$ given by Eq. (10).

$v_{i}(t)$ should be set to be able to perform energy interchange in appropriate directions. Considering energy supply appropriately for energy demand, each AES should perform energy interchange in the direction for adjacent AESs with small sufficiency levels. Namely, $v_{i}(t)$ is set by

$$
v_{i}(t)=\left\{j \mid d_{j}(t)<d_{i}(t), j \in \alpha_{i}\right\} .
$$

The setting of $v_{i}(t)$ with Eq. (12) is simple, but the proposed method with the setting helps diverse situations (e.g., energy shortage and emergency situations) discussed in Section 1. When supplying energy from a macrogrid under energy shortage situations, sufficiency levels of the connected AESs are always 0, and their sufficiency levels are larger than those of other AESs. The sufficiency level of an AES decreases with distance from the connected AESs. When gathering energy to a high priority AES $h$ from other AESs under emergency situations, we set energy demand $\theta_{h}$ to high value, and the sufficiency level of AES $h$ becomes smaller than those of other AESs. The sufficiency level of an AES increases with distance from the high priority AES $h$. Hence, using Eq. (12), each AES can perform energy interchange in the appropriate direction under such situations.

\section{Evaluation}

\subsection{Overview}

In this section, we conduct a simulation experiment in order to evaluate the performance of the proposed method. We first clarify the effectiveness of the directional energy interchange in the proposed method under energy shortage situations and emergency situations. Then, we investigate the performance of the proposed method against the time-varying in energy generation of renewable energy.

In the simulation experiment, we use the $n_{k}$-th nearest neighbor network as mesh network $G$. The $n_{k}$-th nearest neighbor network is generated by the following procedures. Initially, each AES is randomly placed on the two-dimensional plane. Then, each AES selects $n_{k}$ nearest AESs as its adjacent AESs. Note that an AES has adjacent AESs greater than or equal to $n_{k}$. $n_{k}$-th nearest

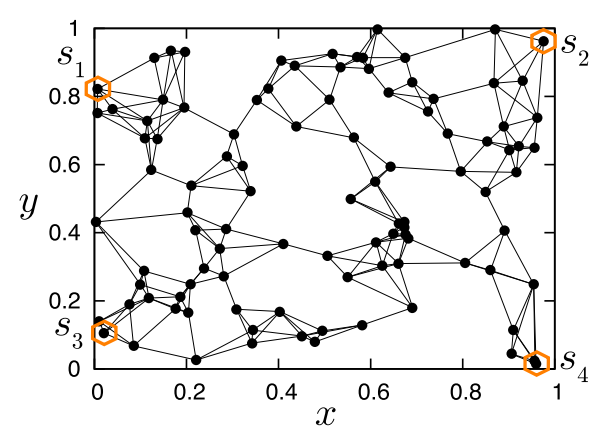

Fig. 7 Examples of $n_{k}$-th nearest neighbor network with $n_{k}=4$ and $n=100$ and external suppliers $s_{1}, s_{2}, s_{3}$, and $s_{4}$.

Table 2 Parameter configuration.

\begin{tabular}{l|c|l}
\hline \hline parameter & symbol & configuration \\
\hline mesh network & $G$ & $n_{k}$-nearest neighbor \\
& & network \\
number of nearest AESs & $n_{k}$ & 4 \\
number of AESs & $n$ & 100 \\
battery capacity of a AES & $\gamma_{i}$ & 100 \\
target remaining battery amount of a AES & $\theta_{i}$ & 50 \\
diffusion coefficient & $\kappa_{\mathrm{diff}}$ & 0.0001 \\
control parameter of MCMC & $\kappa_{\mathrm{MC}}$ & 1,000 \\
advection coefficient & $\kappa_{\mathrm{ad}}$ & 0.1 \\
interval of recalculating $r_{i \rightarrow j}$ & $\Delta T_{r}$ & 1 \\
time period of daylight hours & $T$ & 1,000 \\
coefficient in Eq. (14) & $\kappa_{A}$ & 1.5 \\
ratio in Eq. (15) & $\lambda_{\mathrm{GC}}$ & 2.0 \\
\hline
\end{tabular}

neighbor networks consider geographical dispersion of AESs and wiring cost between AESs. Figure 7 shows an example of 4-th nearest neighbor network for $n=100$, and an example of AESs (hereafter referred to as external suppliers) to be able to connect a macrogrid. In this figure, we denote AESs at the most upper left/right locations and the most lower left/right locations in a $n_{k}$ th nearest neighbor network by $s_{1}, s_{2}, s_{3}$, and $s_{4}$, respectively.

During simulation, each AES transfers own energy according to the MCMC-based ADM using $r_{i \rightarrow j}^{\mathrm{MC}}(t)$ (hereafter referred to as previous mechanism) or using $r_{i \rightarrow j}^{\mathrm{ad}}(t)$ (hereafter referred to as proposed mechanism). The previous mechanism corresponds to the proposed mechanism with $\kappa_{\mathrm{ad}}=0$.

We use the parameter configuration shown in Table 2 as a default parameter configuration. In this paper, we use the same configuration of $\kappa_{\text {diff }}$ and $\kappa_{\mathrm{MC}}$ also used in Ref. [2]. According to the result shown in Ref. [2], as $\kappa_{\text {diff }}$ and $\kappa_{\mathrm{MC}}$ increase, the convergence speed to the equalized state becomes fast. This effect is the same in the previous mechanism and the proposed mechanism. Hence, the configuration of $\kappa_{\text {diff }}$ and $\kappa_{\mathrm{MC}}$ does not affect the conclusion of this paper. If the time period $T$ of daylight hours is 12 hours, the unit time of the simulation is 43.2 seconds when $T=1,000$. This value is calculated by $12 \cdot 3,600 / T$.

\subsection{Fundamental Evaluation}

In order to focus on the effect of the energy interchange on the performance of ADM, we use a static environment model also used in Ref. [2]. In the static environment model, energy generation rate $g_{i}(t)$ of AES $i$ is always equal to energy consumption rate $c_{i}(t)$ of AES $i$ during simulation, and remaining battery amount $b_{i}(t)$ changes with only energy interchange of adjacent AESs $j \in \alpha_{i}$. Hence, we can focus on the difference between the energy interchanges of the previous mechanism and the proposed 


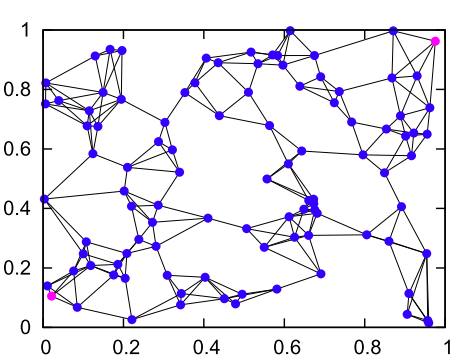

(a) $t=0$

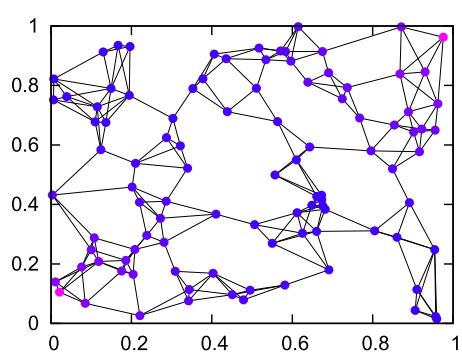

(b) $t=300$

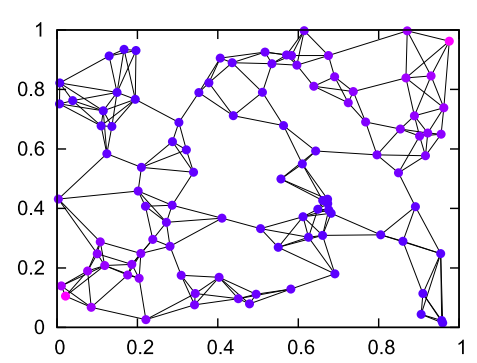

(c) $t=600$

Fig. 9 Geographical distribution of sufficiency levels $d_{i}(t)$ when using the previous mechanism.

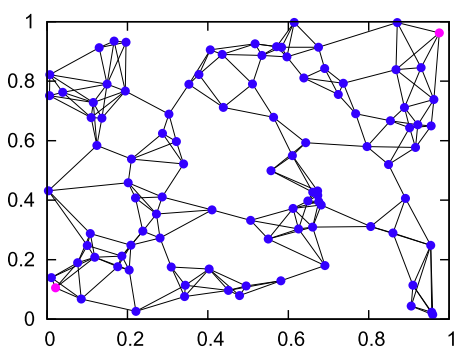

(a) $t=0$

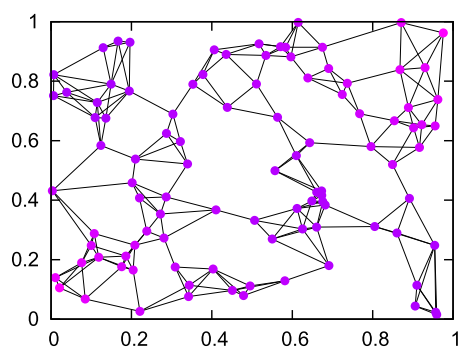

(b) $t=300$

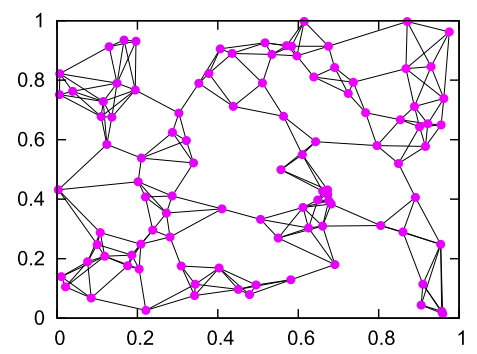

(c) $t=600$

Fig. 10 Geographical distributions of sufficiency levels $d_{i}(t)$ when using the proposed mechanism.

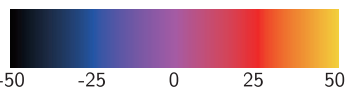

Fig. 8 Color map corresponding to a value for Figs. 9 and 10.

mechanism.

\subsubsection{Result for Energy Shortage Situation}

In this experiment, we choose external suppliers $s_{2}$ and $s_{3}$, which can be only connected to the macrogrid. During the simulation, sufficiency levels $d_{s_{2}}(t)$ and $d_{s_{3}}(t)$ of external suppliers $s_{2}$ and $s_{3}$ are always 0 . For convenience, we show the results when setting initial remaining battery amounts $b_{i}(0)$ of other AESs to 30 for $1 \leq i \leq n$ and $i \neq x\left(x=s_{2}\right.$ and $\left.s_{3}\right)$. Their initial AES sufficiency levels $d_{i}(0)$ are -20 since target remaining battery amount $\theta_{i}$ of all AESs are set to 50. Of course, we also conducted experiment with other initial setting (e.g., randomly setting of $b_{i}(0)$ ), but we obtained the same conclusion for the results shown in this subsection.

First, we visually confirm the effectiveness of the proposed method. Figure 8 illustrates the color map corresponding to a value for visualized results. Figures 9 and $\mathbf{1 0}$ show the geographical distributions of AES sufficiency levels at $t=0,300$, and 600 when using the previous mechanism and the proposed mechanism, respectively. Figure 11 shows time series for statistics (i.e., average and minimum) of AES sufficiency levels when using the previous mechanism and the proposed mechanism. According to these figures, we confirm that the proposed method can quickly energy supply from the external power grid.

The proposed mechanism has control parameter $\kappa_{\mathrm{ad}}$, so we investigate its effect on the speed of the energy supply. As metric for the speed, we use time constant, which is the time required for increasing the minimum of $d_{i}(t)$ to $e^{-1} d_{i}(0) \approx-7.4$. The time constant is a parameter characterizing the convergence speed of a control mechanism. Figure 12 shows time constants for different values of parameter $\kappa_{\mathrm{ad}}$. From this figure, the time constant de-

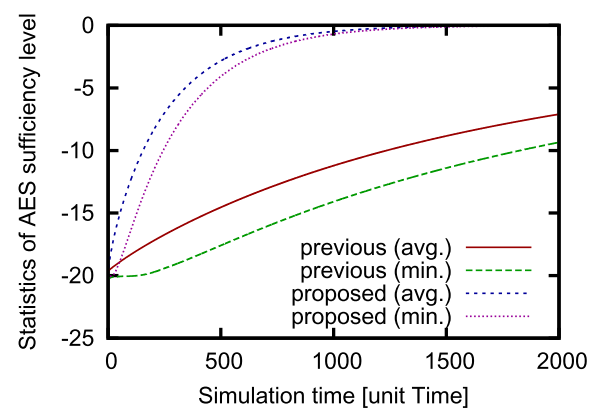

Fig. 11 Time series for statistics of sufficiency levels.

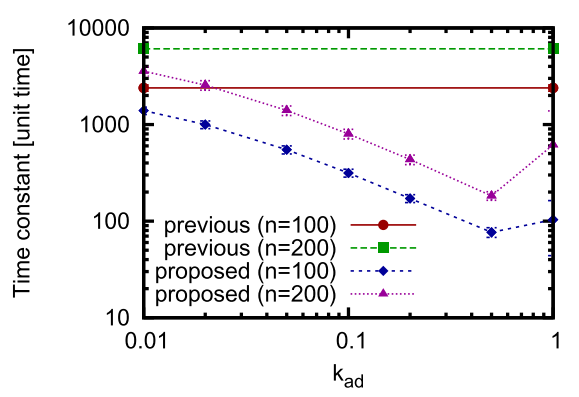

Fig. 12 Time constant for different values of parameter $\kappa_{\mathrm{ad}}$.

creases as $\kappa_{\mathrm{ad}}$ increases except for $\kappa_{\mathrm{ad}}=1$. Note that if $\kappa_{\mathrm{ad}}=1$, the minimum of $d_{i}(t)$ becomes unstable, so the time constant makes no sense. This is caused by the similar reason for the instability of the MCMC-based ADM [2] with too large $\kappa_{\text {diff. }}$. According to the result, if $\kappa_{\mathrm{ad}}$ is sufficiency small, the proposed mechanism works with stability, and it can improve the speed of the previous mechanism. In future work, we should clarify the stability condition for $\kappa_{\mathrm{ad}}$.

We investigate the performance of the proposed mechanism for different levels of energy shortage situation. In this experiment, we obtain the results for different initial AES sufficiency levels of all AESs, and measure times taken to supply energy until 


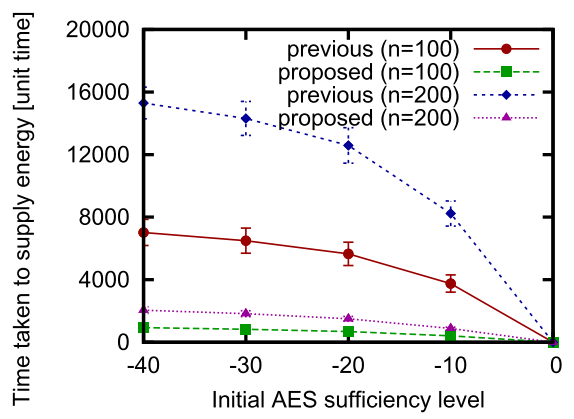

Fig. 13 Time taken to supply energy from the external power grid.

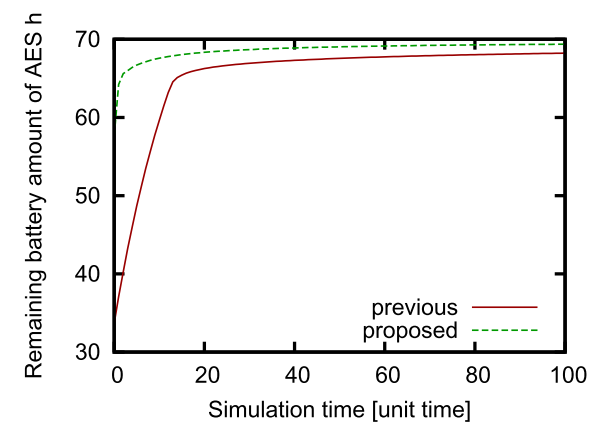

Fig. 14 Time series for the remaining battery amount of AES $h$ with high priority.

$d_{i}(t)= \pm 5 \%$ for all AESs. Figure 13 shows the measured times for different initial AES sufficiency levels of all AESs. According to this result, the improving amount of the proposed method increases as the initial sufficiency level decreases. Hence, the proposed mechanism has high effectiveness for a severe energy shortage situation.

\subsubsection{Result for an Emergency Situation}

Disaster occurs suddenly, and stops equalizing sufficiency levels of AESs halfway. Hence, when disaster occurs, each AES has a different sufficiency level. According to this circumstance, at the start of simulation, we give initial sufficiency level $d_{i}(0)$ of AES $i$ by normal distribution $\mathrm{N}\left(\mu_{s}^{\mathrm{st}}, \sigma_{s}^{\mathrm{st}}\right)$ where $\sigma_{s}^{\mathrm{st}}=0.3 \mu_{s}^{\mathrm{st}}$. We give high target remaining battery amount (i.e., $\theta_{h}=90$ ) to a randomly selected AES $h$ as the high priority AES that means a safe shelter under emergency situation. In this experiment, there are no external suppliers.

Figure 14 shows the time series for the remaining battery amount of AES $h$ with high priority when using the previous mechanism and the proposed mechanism during the simulation. In this simulation, we use $\mu_{s}^{\text {st }}=30$, so the average of initial sufficiency levels $d_{i}(0)$ is about -20 . Note that the average of sufficiency levels is defined by $1 / n \sum_{i=1}^{n} d_{i}(t)$. From this result, the proposed mechanism can fast gather energy to AES $h$. In spite of target remaining battery amount $\theta_{h}=90$ of $\operatorname{AES} h, b_{h}(t)$ is only about 70. Note that $b_{h}(t)=70$ corresponds to $d_{h}(t)=-20$ since we used $\theta_{h}=90$ in this simulation. Since the both mechanisms equalize sufficiency levels of all AES, sufficiency level $d_{h}(t)$ of AES $h$ converges to the average of sufficiency levels. Hence, at the end of the simulation, AES $h$ has only $b_{h}(t)=70$. In order to converge $b_{h}(t)$ to $\theta_{h}$, it is necessary to supply the the shortage energy from the macrogrid to the microgrid.

We then investigate the effect of the location of AES $h$ on the

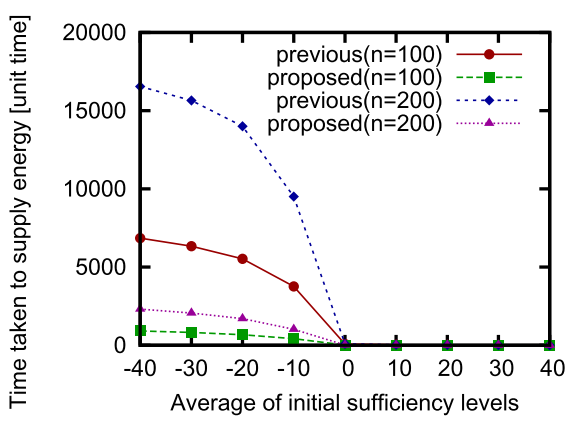

Fig. 15 Average of times taken to supply energy.

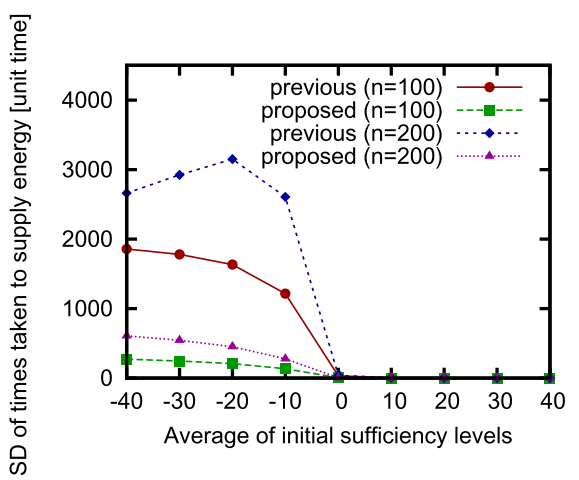

Fig. 16 Standard deviation (SD) of times taken to supply energy.

effectiveness of the proposed mechanism since the speed of the directional energy supply may be affected by the location of AES $h$. Figures $\mathbf{1 5}$ and $\mathbf{1 6}$ show the average and the standard deviation (SD) of times taken to supply energy for different average of initial sufficiency levels, $\mu_{s}^{\text {st }}$, respectively. According to Fig. 15, the proposed mechanism can very fast gather the distributed energy to the high priority AES. From Fig. 16, the SD for the proposed mechanism is always smaller than the previous mechanism. Hence, the proposed mechanism keeps the performance against differences in the location of the high priority AES, compared with the previous mechanism.

Therefore, we can conclude that the proposed mechanism is effective under emergency situations.

\subsection{Advanced Evaluation}

We conduct the simulation experiment with the time-varying characteristic in energy generation of renewable energy. Through the experiment, we investigate the effect of the time-varying characteristic on the performance of the MCMC-based ADM. Of course, we should investigate the effect of other important characteristics (e.g., loss characteristics in energy transformation and transfer), but these investigations will be discussed in the future work due to the space limitation.

In order to clarify on the effect of the time-varying characteristic, we use the simulation model which is made by adding a time-varying model of energy generation to the simulation model used in Section 6.2. We should select the time-varying model carefully. In this paper, we use a minimal time-varying model that has only a few parameters, but can characterize the time variation roughly. With such a minimal time-varying model, we will clarify general properties that can be applied to many situations. If we use a complex time-varying model with many parameters, 


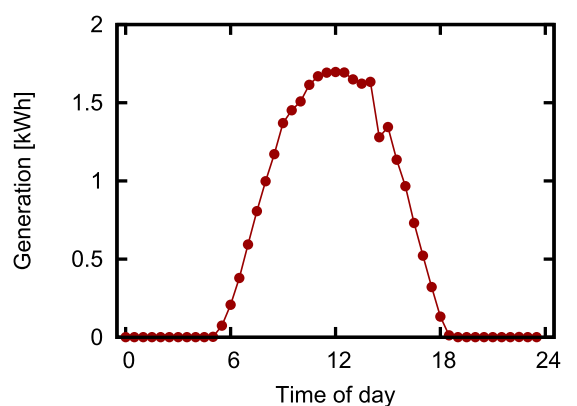

Fig. 17 Time series for the measured amount of energy generation with a PV panel placed in Shiga, Japan.

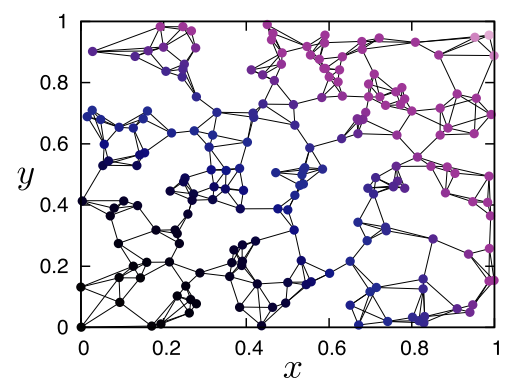

Fig. 18 Geographical distribution of maximum generation rates $A_{i}\left(x_{i}, y_{i}\right)$.

the behavior of the energy generation can be accurate using the parameter fitting with actual data. However, the findings using such a complex model is limited in some situations corresponding to the actual data.

\subsubsection{Time-varying Model}

We introduce the minimal time-varying model of the energy generation used in this paper. Figure $\mathbf{1 7}$ shows the time series of the measured amount of the energy generation with a PV panel placed in Shiga, Japan. Note that the generation data is measured on July 15, 2011. According to the figure, the amount of the energy generation is approximately given by a sine curve. Hence, as a basic model representing the time-varying characteristic of PV panels, the energy generation rate $g_{i}(t)$ of AES $i$ is changing with

$$
g_{i}\left(t ; x_{i}, y_{i}\right)=\left[A_{i}\left(x_{i}, y_{i}\right) \sin \left(\frac{\pi}{T}\left(t-\tau_{i}\right)\right)\right]^{+},
$$

where $A_{i}\left(x_{i}, y_{i}\right)$ is the maximum generation rate of AES $i$ located at $\left(x_{i}, y_{i}\right)$ for $0 \leq x_{i}, y_{i} \leq 1, T$ is the time period of daylight hours, and $\tau_{i}$ is the starting time of the energy generation of AES $i$. In the experiment, $\tau_{i}$ is randomly set to a value $\in[0,1]$. Note that $A_{i}\left(x_{i}, y_{i}\right)$ represents the strength of sunlight at AES $i$. In general, sunlight strength depends on geographical condition and weather, so maximum generation rates $A_{i}\left(x_{i}, y_{i}\right)$ of AESs are usually different values. To investigate the effectiveness of energy interchange, we set $A_{i}\left(x_{i}, y_{i}\right)$ depending on the geographical location of AES $i$. Namely, we use

$$
A_{i}\left(x_{i}, y_{i}\right)=\kappa_{A} \sqrt{\frac{x_{i}^{2}+y_{i}^{2}}{2}},
$$

where $\kappa_{A}$ is the coefficient to adjust the time and spatial average of energy generation rates. In Fig. 18, the color of AES $i$ represents the value of $A_{i}\left(x_{i}, y_{i}\right)$. As $A_{i}\left(x_{i}, y_{i}\right)$ decreases, the color becomes dark. With this setting of $A_{i}$, energy shortage situation will occur

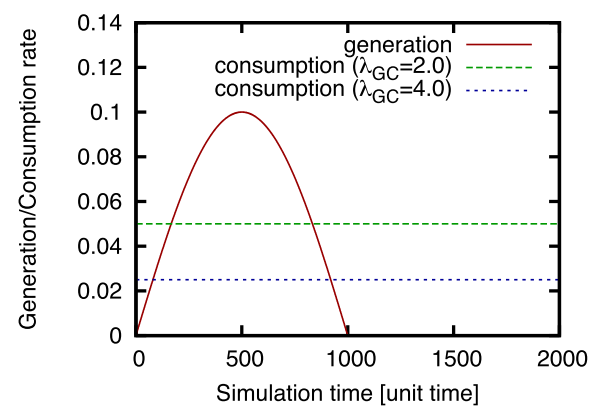

Fig. 19 Time series of the generation rate $g_{i}(t)$ and the consumption rate $c_{i}(t)$ of AES $i$ in the time-varying model with $T=1,000$ and $A_{i}=0.1$.

at AESs near the origin $(0,0)$. This setting of $A_{i}$ supposes that local heavy rain is caused around $(0,0)$. Our ADM should transfer surplus energy to the AESs with energy shortage from AESs near the point $(1,1)$.

To focus on the effect of the time variation in energy generation, energy consumption rate $c_{i}(t)$ is given by the constant function

$$
c_{i}(t)=\frac{\bar{A}}{\lambda_{\mathrm{GC}}},
$$

where $\lambda_{\mathrm{GC}}$ is the ratio of the consumption rate by $\bar{A}$, which is the time and spatial average of generation rates $g_{i}\left(t ; x_{i}, y_{i}\right)$ for $1 \leq i \leq n$. Figure 19 shows the time series of the generation rate $g_{i}(t)$ for $T=1,000$ and $A_{i}=0.1$ and the consumption rate $c_{i}(t)$ for $\lambda_{\mathrm{GC}}=2.0$ and 4.0 .

Since the location $\left(x_{i}, y_{i}\right)$ of AES $i$ follows the uniform distribution in the $n_{k}$-th nearest neighbor network, $\bar{A}$ is estimated by

$$
\begin{aligned}
\bar{A} & =\frac{1}{n} \sum_{i=1}^{n}\left(\int_{0}^{2 T} g_{i}\left(t ; x_{i}, y_{i}\right) \mathrm{d} t\right) \\
& \simeq \int_{0}^{1} \int_{0}^{1}\left(\int_{0}^{2 T} g_{i}(t ; x, y) U(x, y) \mathrm{d} t\right) \mathrm{d} x \mathrm{~d} y \\
& =\frac{1}{3} \kappa_{A}\left(1+\frac{\log (1+\sqrt{2})}{\sqrt{2}}\right) \approx 0.54 \kappa_{A},
\end{aligned}
$$

where $U(x, y)$ is the two-dimensional uniform distribution for $0 \leq x, y \leq 1$. By substituting the above approximated $\bar{A}$ into Eq. (15), we obtain

$$
c_{i}(t)=\bar{C} \approx \frac{0.54 \kappa_{A}}{\lambda_{\mathrm{GC}}}
$$

where $\bar{C}$ is the time and spatial average of $c_{i}(t)$ for $1 \leq i \leq n$. According to Eqs. (16) and (17), $\bar{A}$ and $\bar{C}$ are approximately proportional to $\kappa_{A}$, and the difference between them is determined with $\lambda_{\mathrm{GC}}$. If $\lambda_{\mathrm{GC}}$ is a small value, the consumption rates are relatively large compared with the generation rates, and the microgrid will face a severe energy shortage situation without the energy supply from an external power grid. In this experiment, we investigate the performance of our ADM for different values of $\lambda_{\mathrm{GC}}$ in order to clarify the effectiveness of our ADM for several situations with different levels of energy shortage situation.

At the start of simulation, we give initial remaining battery amount $b_{i}(0)$ of AES $i$ by normal distribution $N(30,10)$. We terminate the simulation at time $t=2 T$. 


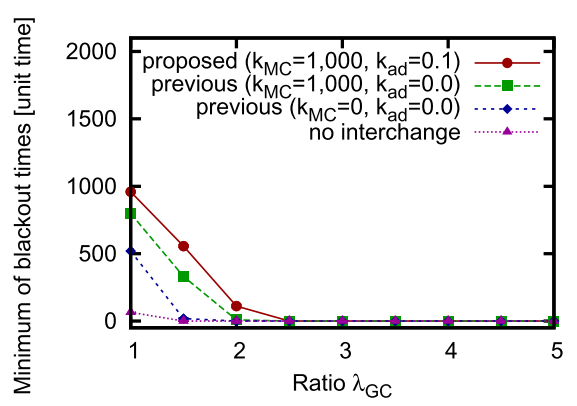

(a) minimum of blackout times

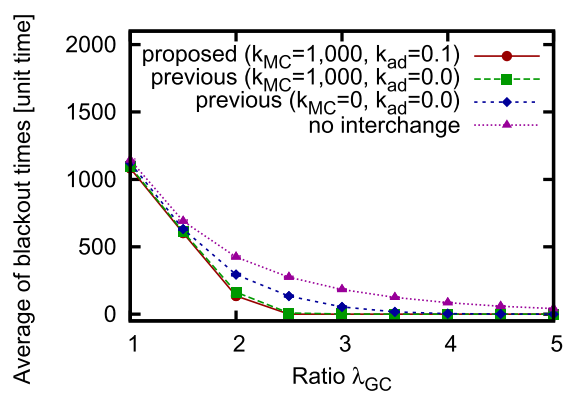

(b) average of blackout time

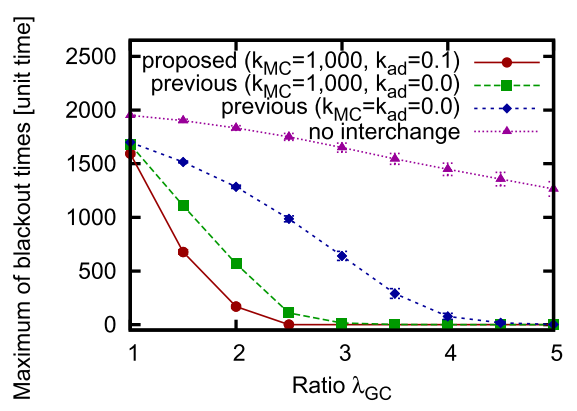

(c) maximum of blackout times

Fig. 20 Blackout times for different ratio $\lambda_{\mathrm{GC}}$.

In the experiment, we measure blackout times of each AES. The blackout time of AES $i$ is defined as the time period during remaining battery amount $b_{i}(t)=0$. We discuss how our ADM can reduce blackout times using energy interchange among AESs.

\subsubsection{Result for the Previous Mechanism}

We have not evaluated the performance of the previous mechanism with any time-varying model. Hence, we first investigate the effect of the time-varying in energy generation on the previous mechanism. In this experiment, we compare results for $\kappa_{\mathrm{MC}}=0$ and $\kappa_{\mathrm{MC}}>0$ to clarify the effectiveness of the non-linear diffusion effect of the MCMC term (i.e., the term with $\kappa_{\mathrm{MC}}$ in the right-hand side of Eq. (3)).

Figures 20 (a) through (c) show the minimum, the average, and the maximum of blackout times for different ratio $\lambda_{\mathrm{GC}}$, respectively. In these figures, we also show the results without energy interchange (no interchange) and the proposed mechanism as reference. According to these figures, our ADM with $\kappa_{\mathrm{MC}}=1,000$ can reduce the average and the maximum of blackout times, but increase the minimum of blackout times. This is because the previous mechanism performs energy interchange so that the variance of remaining energies is fast decreased.

We assume that the proposed mechanism is used in an energy

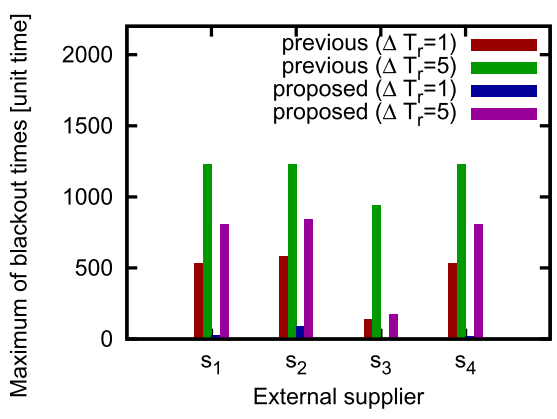

Fig. 21 Maximum of blackout times for different external suppliers.

shortage situation and a normal situation. Hence, we need to investigate the performance of the proposed mechanism in normal situations. In the evaluation of this section, the energy is not supplied from the macrogrid. The result shown in Fig. 20 helps to know the performance in a normal situation. According to the result, the proposed mechanism has the same effect in the previous mechanism with $\kappa_{\mathrm{MC}}=1,000$ and $\kappa_{\mathrm{ad}}=0$. Therefore, the proposed mechanism seems to also work well in the normal situation.

\subsubsection{Result for the Proposed Mechanism}

We then investigate the effect of the generation time-varying on the proposed mechanism by comparing the results with the previous mechanism $\left(\kappa_{\mathrm{ad}}=0\right)$ and the proposed mechanism $\left(\kappa_{\mathrm{ad}}=0.1\right)$. In this experiment, we select an external supplier from $s_{1}, s_{2}, s_{3}$, or $s_{4}$ shown in Fig. 7 .

Figure 21 shows the maximum of blackout times for different external suppliers. At the start of the simulation, the energy generation amount for each AES is smaller than the energy consumption amount for each AES. Hence, most of the blackout occurs at the start of the simulation. The time to supply energy with the proposed mechanism cannot be 0 . Hence, there is a limitation for the speed of the energy supply with the proposed mechanism. According to this figure, (a) the location of external supplier $s_{3}$ is the best to fill the shortage energy in the microgrid because external supplier $s_{3}$ is nearest to the energy shortage area where maximum generation rates $A_{i}$ of AESs are low, and (b) the proposed mechanism with $\Delta T_{r}=1$ can prevent blackout in the microgrid. Hence, the proposed mechanism with sufficiently small $\Delta T_{r}$ is effective against the time variation in energy generation. Moreover, in order to avoid the blackout, $\Delta T_{r}$ should be sufficiently small value, and the external supplier should be placed near AESs that severe energy shortage is expected.

\section{Conclusion and Future Work}

In this paper, toward resilient microgrids, we proposed a method of directional energy interchange used in the MCMCbased ADM. We first designed a method of the directional energy interchange to be able to quickly transfer energy in an appropriate direction on the basis of the advection-diffusion phenomenon observed in physics. Then, we investigated the performance of the proposed method through a simulation experiment considering energy shortage and emergency situations. We clarified that the proposed method performs fast directional energy interchange under these situations. Hence, we conclude that the MCMC-based ADM with the proposed method helps diverse sit- 
uations. Moreover, we evaluated the effectiveness of our ADM against the fluctuation of energy generation with PV panels. The simulation results also showed the effectiveness of the proposed method under the generation fluctuation.

To realize the proposed method in a practical electricity system, we should further investigate the performance of the proposed method. The division into AESs improves the reliability and scalability of microgrids, but generates energy loss by energy interchange among AESs. In this paper, we ignored such energy loss in the evaluation. We should clarify the effect of the energy loss on the effectiveness of the proposed method. Moreover, we should investigate the performance with considering realistic characteristics (e.g., the limitation of rate-of-change in battery charging).

\section{References}

[1] CERTS: Integration of Distributed Energy Resources: The CERTS MicroGrid Concept, available from 〈https://certs.lbl.gov/publications/ integration-distributed-energy $\rangle$ (accessed 2017-08-08).

[2] Sakumoto, Y. and Taniguchi, I.: Autonomous decentralized mechanism for energy interchanges with accelerated diffusion based on MCMC, IEICE Trans. Fundamentals of Electronics Communications and Computer Sciences, Vol.E98-A, No.7, pp.1504-1511 (2015).

[3] NEXT KRAFTWERKE: Virtual Power Plant, available from 〈https://www.next-kraftwerke.com/〉 (accessed 2019-08-07).

[4] Energy2Market: Aggregation and Energy Trading - with e2m, available from 〈https://www.e2m.energy/en/start-en.html (accessed 201908-07).

[5] Enernoc: The World Leader in Energy Intelligence, available from $\langle$ https://www.enernoc.com/index.php (accessed 2019-08-07).

[6] The Clean Coalition: Community Microgrid, available from 〈http://www.clean-coalition.org/our-work/community-microgrids/〉 (accessed 2017-08-08).

[7] LO3 ENERGY: Brooklyn Microgrid, available from 〈http://brooklynmicrogrid.com/〉 (accessed 2017-08-08)

[8] Presidential Policy Directive (PPD-21): Critical Infrastructure Security and Resilience, available from $\langle$ http://www.whitehouse.gov/thepress-office/2013/0/12/presidential-policy-directive-criticalinfrastructure-security-and-resil $>$ (accessed 2017-08-08).

[9] Sakumoto, Y. and Taniguchi, I.: Proposal for Fast Directional Energy Interchange Used in MCMC-Based Autonomous Decentralized Mechanism toward Resilient Microgrid, Proc. 2016 Design, Automation and Test in Europe (DATE 2016), pp.237-240 (2016).

[10] Sakumoto, Y. and Taniguchi, I.: Evaluation of MCMC-Based Autonomous Decentralized Mechanism of Energy Interchange in Practical Scenario with Generation Fluctuation, Proc. 22nd IEEE International Conference on Emerging Technologies And Factory Automation (ETFA 2017), pp.1-5 (2017).

[11] Abe, R., Taoka, H. and McQuilkin, D.: Digital grid: Communicative electrical grids of the future, IEEE Trans. Smart Grid, Vol.2, No.2, pp.399-410 (2011).

[12] Taniguchi, T. and Yano, S.: Decentralized trading and demand side response in inter-intelligent renewable energy network, Proc. IEEE Joint 6th International Conference on Soft Computing and Intelligent Systems (SCIS 2012) and 13th International Symposium on Advanced Intelligent Systems (ISIS 2012), pp.645-650 (2012).

[13] Go Electric Inc.: Go Electric Inc. Projects, available from $\langle$ http://www.goelectricinc.com/projects.html〉 (accessed 2017-08-08).

[14] Microgrid Projects: Sendai Microgrid, available from 〈http://microgridprojects.com/microgrid/sendai-microgrid/〉 (accessed 2017-08-08).

[15] Hirose, K., Shimakage, T., Reilly, J.T. and Irie, H.: The Sendai Microgrid Operational Experience in the Aftermath of the Tohoku Earthquake: A Case Study, NEDO Microgrid Case Study (2013).

[16] Khodaei, A.: Resiliency-oriented microgrid optimal scheduling, IEEE Trans. Smart Grid, Vol.5, No.4, pp.1584-1591 (2014).

[17] Marnay, C., Aki, H., Hirose, K., Kwasinski, A., Ogura, S. and Shinji, T.: Japan's pivot to resilience: How two microgrids fared after the 2011 earthquake, IEEE Power and Energy Magazine, Vol.13, No.3, pp.44-57 (2015).

[18] Colson, C., Nehrir, M. and Gunderson, R.: Distributed multi-agent microgrids: A decentralized approach to resilient power system selfhealing, Proc. 4th International Symposium on Resilient Control Sys- tems (ISRCS), pp.83-88, IEEE (2011).

[19] Chen, C., Wang, J., Qiu, F. and Zhao, D.: Resilient distribution system by microgrids formation after natural disasters, IEEE Trans. Smart Grid, Vol.7, No.2, pp.958-966 (2016).

[20] Ren, W., Beard, R.W. and Atkins, E.M.: A survey of consensus problems in multi-agent coordination, Proc. American Control Conference, pp.1859-1864, IEEE (2005).

[21] Olfati-Saber, R., Fax, J.A. and Murray, R.M.: Consensus and cooperation in networked multi-agent systems, Proc. IEEE, Vol.95, No.1, pp.215-233 (2007).

[22] Hastings, W.: Monte Carlo sampling methods using Markov chains and their applications, Oxford Journal Biometrika, Vol.57, No.1, pp.97-109 (1970)

[23] Newman, M.E. and Barkema, G.T.: Monte Carlo methods in statistical physics, Oxford University Press (1999).

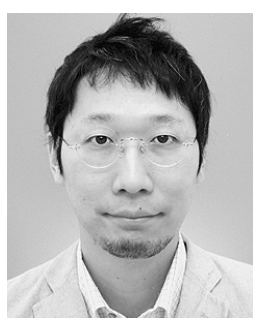

Yusuke Sakumoto received M.E. and Ph.D. degrees in the Information and Computer Sciences from Osaka University in 2008 and 2010, respectively. He is currently an associate professor at Kwansei Gakuin University, Japan. His research work is in the area of communication network, electricity network, and social network. He is a member of IEEE, IEICE and IPSJ.

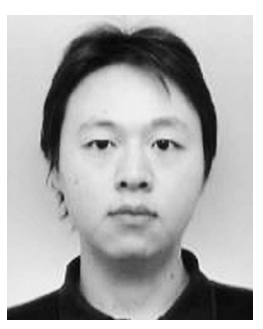

Ittetsu Taniguchi received B.E., M.E., and Ph.D. degrees from Osaka University in 2004, 2006, and 2009, respectively. From 2007 to 2008, he was an international scholar at Katholieke Universiteit Leuven (IMEC), Belgium. In 2009, he joined the College of Science and Engineering, Ritsumeikan University as an assistant professor, and became a lecturer in 2014. In 2017, he joined the Graduate School of Information Science and Technology, Osaka University as an associate professor. His research interests include system level design methodology, and combinatorial optimization problems. He is a member of IEEE, ACM, IEICE, and IPSJ. 